\title{
El apoyo telefónico aumenta las tasas de rastreo de cáncer en mujeres de bajos recursos
}

Dietrich MD et al. Ann Inter Med. 2006; 144:563-571.

\section{Objetivo}

Evaluar el impacto del apoyo telefónico para aumentar las tasas de rastreo de cáncer de mama, cuello uterino y colon en mujeres de bajos recursos.

\section{Diseño}

Ensayo clínico aleatorizado controlado, realizado desde Noviembre de 2001 hasta Abril de 2004

\section{Lugar}

11 centros comunitarios de salud de Nueva York, EEUU.

\section{Pacientes}

Se aleatorizaron 1413 mujeres entre 50 y 69 años que no habían cumplido con las pautas de frecuencia recomendadas para rastreo de cáncer de mama, cuello uterino o colon.

\section{Intervención}

Durante 18 meses las mujeres asignadas al grupo intervención $(n=706)$ recibieron un promedio de 4 llamadas de agentes especialmente entrenadas en estrategias para fomentar la conciencia de necesidad y facilitar el acceso a pruebas de rastreo (ver cuadro 1). Se obtuvieron datos al final del seguimiento del $99 \%$ de las pacientes y $91 \%$ del grupo intervención recibió al menos una llamada telefónica. Las asignadas al grupo control $(n=707)$ recibieron los cuidados habituales.

Cuadro 1. Estrategias para fomentar la conciencia de necesidad y facilitar el acceso al rastreo de cáncer.

\section{Estrategias Utilizadas}

\section{- Fomentar la conciencia de necesidad:}

a- Proveer información sobre los beneficios del rastreo.

b- Envío de recordatorios postales sobre la frecuencia recomendada de rastreo.

c- Envío de material informativo.

\author{
- Facilitar el acceso: \\ a- Facilitación de turnos. \\ b- Recordatorios de turnos. \\ c- Consejo sobre formas de acceso al sistema de salud.
}

\section{Medición de resultados principales.}

Proporción de mujeres con mamografía, Papanicolau y rastreo de cáncer de colon actualizado al finalizar la intervención.

El análisis fue por intención de tratar.

\section{Resultados principales.}

Tabla 1. Incidencia de rastreo de cada tipo de cáncer y diferencias entre los grupos

\begin{tabular}{|c|c|c|c|}
\hline & $\begin{array}{l}\text { Grupo Intervención } \\
\text { (n=696) }\end{array}$ & $\begin{array}{l}\text { Con estribos } \\
\text { Media (DS) }\end{array}$ & Diferencia (IC 95\%) \\
\hline \multicolumn{4}{|l|}{ Mamografía } \\
\hline Al comienzo & $58 \%$ & $60 \%$ & NS \\
\hline Al final & $68 \%$ & $58 \%$ & $10 \%(5 \%$ a $15 \%)$ \\
\hline Diferencia comienzo-final & $10 \%(5 \%$ a $15 \%)$ & NS & $12 \%(6 \%$ a $19 \%)$ \\
\hline \multicolumn{4}{|l|}{ Papanicolaou } \\
\hline Al comienzo & $71 \%$ & $70 \%$ & NS \\
\hline Al final & $78 \%$ & $70 \%$ & $8 \%(3 \%$ a $12 \%)$ \\
\hline Diferencia comienzo-final & $7 \%(3 \%$ a $11 \%)$ & NS & $7 \%(1 \%$ a $12 \%)$ \\
\hline \multicolumn{4}{|l|}{ Rastreo de cáncer de colon } \\
\hline Al comienzo & $39 \%$ & $39 \%$ & NS \\
\hline Al final & $63 \%$ & $50 \%$ & $13 \%(8 \%$ a $18 \%)$ \\
\hline Diferencia comienzo-final & $24 \%(20 \%$ a $29 \%)$ & $11 \%(8 \%$ a $16 \%)$ & $13 \%(7 \%$ a $19 \%)$ \\
\hline
\end{tabular}

\section{Conclusiones}

El apoyo telefónico aumentó significativamente la tasa de rastreo de cáncer de mama, cuello uterino y colon, en mujeres de bajos recursos.

Fuente de financiamiento: Instituto Nacional del Cáncer de Estados Unidos.

\section{Comentario}

Las mujeres de bajos recursos son un grupo vulnerable, con mayor riesgo de diagnóstico de cánceres en estadios más avanzados cuando el tratamiento es sumamente costoso y menos exitosodebido principalmente a la baja adherencia a las maniobras recomendadas de rastreo ${ }^{1}$.

Este estudio demuestra que el apoyo telefónico puede mejorar las tasas de rastreo de los 3 tipos de cáncer más frecuentes en esta población, con una estrategia sencilla como el contacto telefónico. Si bien uno se ve tentado a ensayar esta estrategia en nuestro país, algunos puntos deberían ser considerados en primer lugar. Las pacientes de bajos recursos de este estudio pertenecían a una población que recibía sus cuidados regulares en una clínica de la comunidad desde al menos 6 meses atrás, que mantenía una historia clínica y que no tenía planes de cambiar de centro de salud en los siguientes 15 meses. Esto difiere sustancialmente de la modalidad de atención de nuestra población de bajos recursos, al igual que la disponibilidad de teléfono y hasta de Internet, ya que en la intervención también se incluyó el envío de correos electrónicos a algunas pacientes. De todos modos, la intervención del estudio como promoción de la salud mediante un sistema del tipo "call center", puede ser fácilmente implementado, y puede ser especialmente útil en sistemas o incluso centros de salud que ofrecen continuidad y longitudinalidad en el cuidado de su población.

El estudio demostró un impacto moderado durante los 18 meses que duró la intervención. Como siempre en este tipo de estudios, una vez demostrada la utilidad, es necesario incorporarla a la práctica cotidiana para garantizar que el efecto se mantenga en el tiempo.

\section{Conclusiones del comentador}

El apoyo telefónico es una estrategia interesante para aumentar las tasas de rastreo de los cánceres más frecuentes en las mujeres. Esta intervención requiere que los centros mantengan historias clínicas actualizadas y que la población mantenga su fuente regular de atención de salud a lo largo del tiempo.

Ver glosario*

Plinio Gaglio [ Unidad de Medicina Familiar y Preventiva del Hospital Italiano de Buenos Aires. ]

Recibido el 25 de septiembre de 2006 y aceptado el 5 de octubre de 2006.

Gaglio P. El apoyo telefónico aumenta las tasas de rastreo de cáncer en mujeres de bajos recursos Evid. Actual. Práct. Ambul. 9(6); 167 Nov-Dic. 2006. Comentado de: Dietrich MD; Tobin PhD; Cassells MPH. Telephone care managment to improve cancer screening among low-income women. Ann Inter Med. 2006;144:563-571. PMID: 16618953 